\title{
THE U.S. NAVAL OBSERVATORY PARALLAX PROGRAM
}

\author{
K. Aa. STRAND, R. S. HARRINGTON, and C. C. DAHN \\ U.S. Naval Observatory, Washington, D.C., U.S.A.
}

\begin{abstract}
The U.S. Naval Observatory program on trigonometric stellar parallaxes with the 61 -in. astrometric reflector has been in progress since 1964. To date 201 definitive and 8 preliminary negligible parallaxes have been published, including the UBV photometry of these stars. Data for an additional 35 stars are still unpublished.

The mean error in position for an image of unit weight is $1.2 \mu$ or 0.016 , of which $0.8 \mu$ originates from the measuring error of the automatic measuring machine.

An error of the parallax of 0.004 can usually be obtained with 40 or fewer plates and with an average parallax factor of 0.7 .

A statistical investigation of the derived parallaxes shows that they are free from significant internal systematic errors.
\end{abstract}

\section{Introduction}

The U.S. Naval Observatory (USNO) parallax program with the 61-in. Astrometric Reflector at the USNO Flagstaff Station in Arizona has been in operation since April 1964; to date some 25000 plates have been obtained. Since the telescope and the SAMM automatic measuring machine have been described elsewhere (Strand, 1971) no further description will be made here. The thorough testing of the telescope in the initial stage (Hoag et al., 1967) showed it to be a highly stable instrument, both mechanically and optically. Its operation over the past 9 years has corroborated this.

We will briefly discuss the following at this time:

(1) The results of the program.

(2) The accuracy of the astrometric data.

(3) The photometric program.

(4) An analysis of the lower main sequence and the degenerate branch in the Hertzsprung Russell Diagram.

\section{The Results of the Program}

Two catalogs of stellar parallaxes have been published. The first catalog (Riddle, 1970) contains 100 parallaxes, while the second catalog (Routly, 1972) has 109 parallaxes. The third catalog, unfortunately, is not as near completion as originally scheduled because of persistent unfavorable observing conditions at Flagstaff during a six months period. Beginning with the second catalog, preliminary parallaxes and proper motions have been published for stars which show near zero parallaxes. These data are based upon limited, but sufficient, plate material which has established that further effort on these stars is not likely to produce significant parallax information. This procedure seems justified since the program is aimed at obtaining parallaxes of nearby degenerate and late main sequence stars without attempting to establish statistically significant data in regard to distribution of these stars in space. In all, 
seven stars within $10 \mathrm{pc}$ and with no previously published parallaxes have been found. Two stars with preliminary parallaxes larger than 0".2 (Riddle et al., 1971; Dahn et al., 1972) are currently on the program for completion as are 8 stars with preliminary parallaxes between 0 .' 1 and 0 .'2.

\section{The Accuracy of the Data}

The mean error in position for an image on the plate of unit weight was found to be $1.2 \mu$ or 0 ".016. This error depends upon the average assigned weight of the plates in the individual series and is a somewhat arbitrary value in that a systematic increase or decrease in weight would correspondingly raise or lower this value. A further investigation of the weighting scheme (which depends upon an estimate of the quality of the image, made at the time of measurement) has shown (Harrington) that all images, regardless of weight on the present weighting scheme indicate the same mean errors of position. The average absolute parallax factor in $x$ is 0.68 . Typically a parallax based upon 36 plates has a mean error of 0.004 .

In addition to these conventional expressions for the internal accuracy of the parallaxes, other tests were devised (by Harrington). One test investigated the number of plates required in a series before a parallax levelled off to its final value. This was found to be approximately 25 plates. It therefore appears that the chosen number of 36 plates in a series provides sufficient margin for establishing a stable final value for a parallax.

Another test consisted of assuming a parallax and proper motion for various program stars and attempting to re-derive those values. The position of a star was calculated for each of the times plates were taken in an actual parallax series, using the adopted parallax and proper motion and adding a random error from a normal distribution with a standard deviation of $1.2 \mu$ (equal to the positional accuracy of SAMM). From the positions thus obtained, a fictitious parallax was calculated which, in all cases, differed only slightly from the assumed parallax. This procedure was repeated 5 times for each of the stars in the second catalog. The residuals of the fictitious parallaxes, compared with the assumed parallax, were normally distributed with a mean error of 0.004 , equal to the observed internal mean error of USNO parallax determinations. From this it may be concluded that the USNO parallaxes are free from serious internal systematic errors.

The present reduction program provides, in addition to the parallax and proper motion with their mean errors of the parallax star, the preliminary values of these quantities for the comparison stars as a safeguard against having a comparison star with an appreciable parallax vitiating the final value of the parallax of the star in question. No attempt has been made to use these parallaxes to establish an external mean error of the derived parallaxes, which they obviously cannot produce.

In regard to the external errors of the derived parallaxes, using the conventional method of comparing ours with those derived at other observatories. the total number of parallaxes in common is still insufficient to establish these errors (system- 
atic as well as random errors). A very preliminary investigation of this kind (Gliese, 1972) seems to indicate that the USNO parallaxes differ by an amount of +0 ".002 \pm 0.003 from the Jenkins General Catalog of Trigonometric Parallaxes and that they have an external mean error of \pm 0.0085 .

\section{The Photometric Program}

The photometric program, instituted for the purpose of supplementing the astrometric data with photoelectrically determined magnitudes and colors, continues with both the 61-in. (J. B. Priser) and 40-in. reflectors (C. C. Dahn and H. H. Guetter) at the Flagstaff Station. However, two modifications to the original program have been made since June 1971. They are:

(1) $U$ bandpass measures of the lower main sequence stars have been discontinued; and

(2) $V-I$ color indices on the photometric system of Kron and Mayall (1960) are

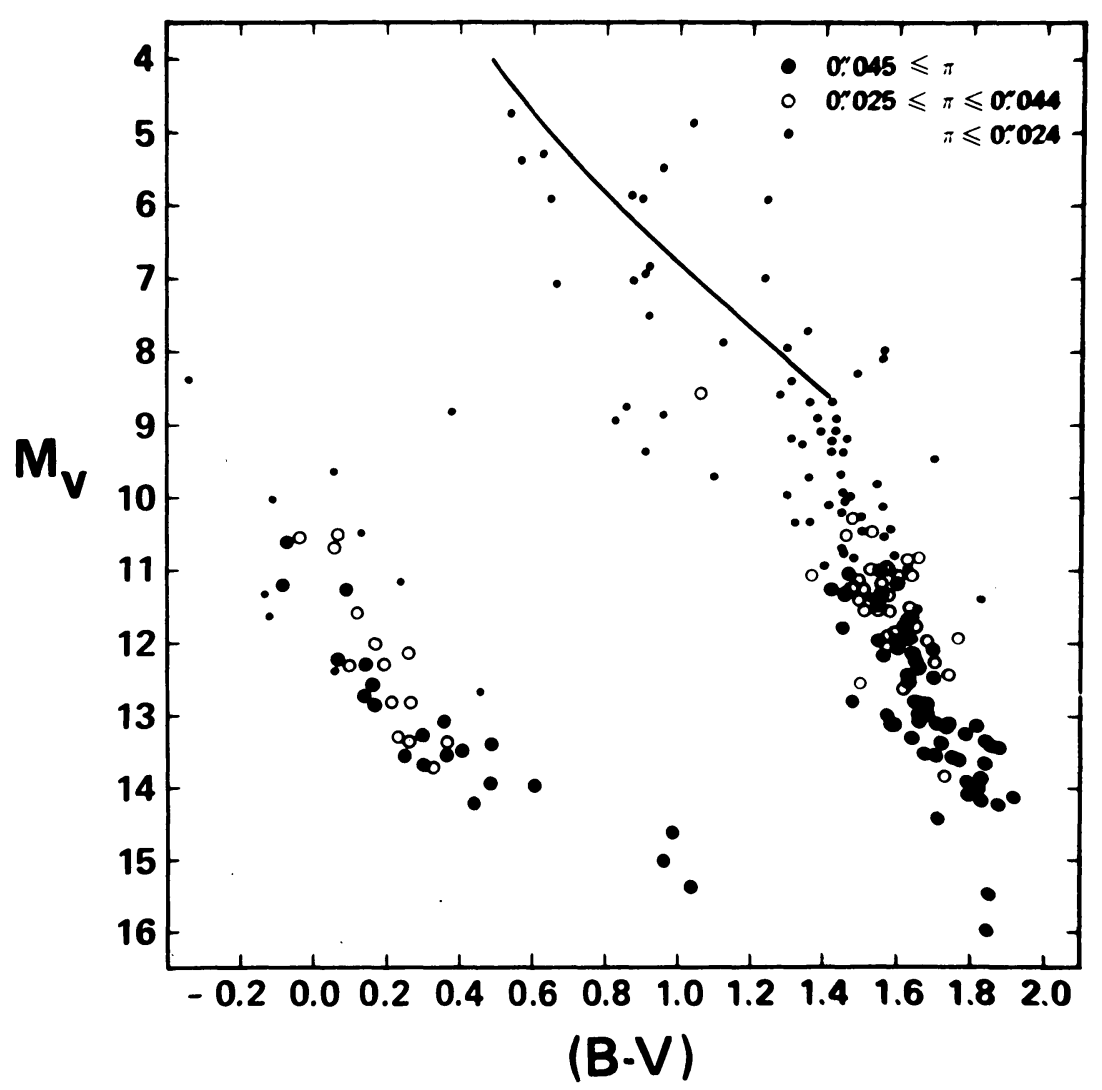

Fig. 1. The HR diagram for stars for which parallaxes and photometric data were obtained at the U.S. Naval Observatory and published in its first two catalogs. 
now being measured for the red dwarf stars by Dahn and Guetter with the 40 -in. Ritchey-Chrétien reflector.

The justification for making these changes is twofold:

(1) The faintness of the red dwarf program stars in the $U$ bandpass precludes obtaining $U-B$ color indices with sufficient accuracy to warrant the effort. For example, of the approximately 70 red dwarf stars for which astrometric and photometric results were reported in the 'Second Catalog of Trigonometric Parallaxes of Faint Stars' (Routly, 1972), 60\% were fainter than $U=16.0$ and $30 \%$ were fainter than $U=17.0$.

(2) The superiority of a color index employing a bandpass in the near infrared over one with bandpasses in the blue is well recognized for red dwarf stars.

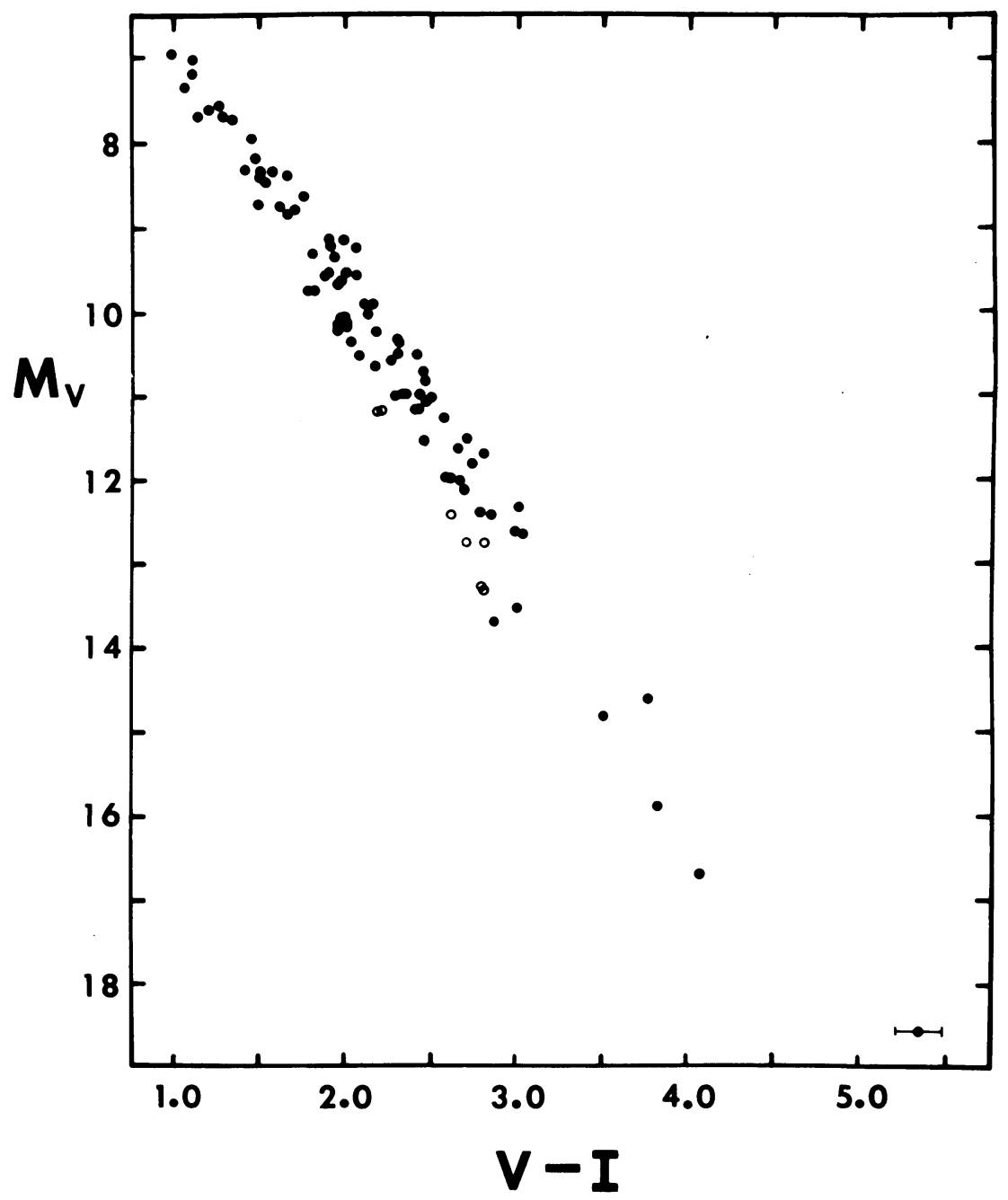

Fig. 2. $M_{v}, V-I$ color magnitude diagram of red dwarfs with trigonometric parallaxes larger than 0.045. Colors were determined by C. C. Dahn and H. H. Guetter at the U.S. Naval Observatory, Flagstaff Station. 


\section{An Analysis of the Lower Main Sequence and the Degenerate Branch in the Hertzsprung-Russell Diagram}

In the Figure 1 is shown a HR diagram based entirely upon the USNO parallaxes published in the two catalogs previously referred to. The $B-V$ colors for the majority of the stars are also from the USNO program. It should be noted that the data have made a substantial contribution to the establishment of the lower main sequence and the degenerate branch.

Figure 2 shows the $M_{v}, V-I$ color magnitude diagram obtained in the previously mentioned $V-I$ photometry by Dahn and Guetter. The selected stars all have well determined published parallaxes, and each data point is based upon a minimum of 3 independent measures of $V-I$. Van Biesbroeck's star based upon a Kron (1958) value is included with an error bar. Open circles in the diagram identify stars classified as subdwarfs on the Mt Wilson spectroscopic system by Joy (1947).

Data obtained for 45 red stars with published USNO parallaxes larger than 0.05 are shown as crosses in the next $M_{v}, V-I$ diagram, Figure 3 . They clearly provide a significant contribution towards delineating the lower main sequence. There are at least an additional 25 stars in the USNO program in the same category as the ones shown, for which only preliminary results are available. There are indications that about 7 of these will turn out to be intrinsically fainter than $M_{v}=14$.

We shall next briefly discuss the delineation in regard to luminosity and color distribution of the Eggen (1969) subluminous stars, which he defines as having $U-V \overline{>} 0.0$ and are at least 2.5 below the main sequence in the $M_{v}$ vs $U-V$ color magnitude diagram. Figure 4 is reproduced from the Eggen-Greenstein (1965) paper and summarizes the observational material available at that time. The dotted line has been added to delineate the Eggen's subluminous designation. The individual stars are identified by their Lowell proper motion survey numbers.

The current status of the stars in question as determined from published parallaxes and from unpublished preliminary USNO parallaxes is shown in Figure 5. Of the 15 stars identifiable with the red degenerates, USNO parallaxes have been published for 6 and preliminary parallaxes are now available for an additional 7 of these stars.

We shall at this time only discuss the three stars lying above the red degenerate branch, G14-24, HG7-138 and G5-28. The USNO parallax for G14-24 is 0".011 \pm 0 ".004 (m.e.) contradicting the Cape parallax of 0".074, and supports the spectroscopic classification as being a weaklined $\mathrm{K}$ subdwarf (thereby permitting Greenstein and Eggen (1966) to retain their spectroscopic badges). In the same manner the very preliminary USNO parallax $(\pi=-0.008 \pm 0.009$ (m.e.)) for HG7-138 suggests it is not a member of the Hyades cluster.

G5-28 with a USNO parallax of +0 ".020 \pm 0.003 appears to definitely depart from the other wise well defined branch. (If the star should conform to the degenerate branch it should have a parallax of 0.072 , while a parallax of 0.005 would place the star among the subdwarfs.) In view of the size of the observed parallax the latter is the more likely case. With this exception we conclude on the basis of the USNO data 


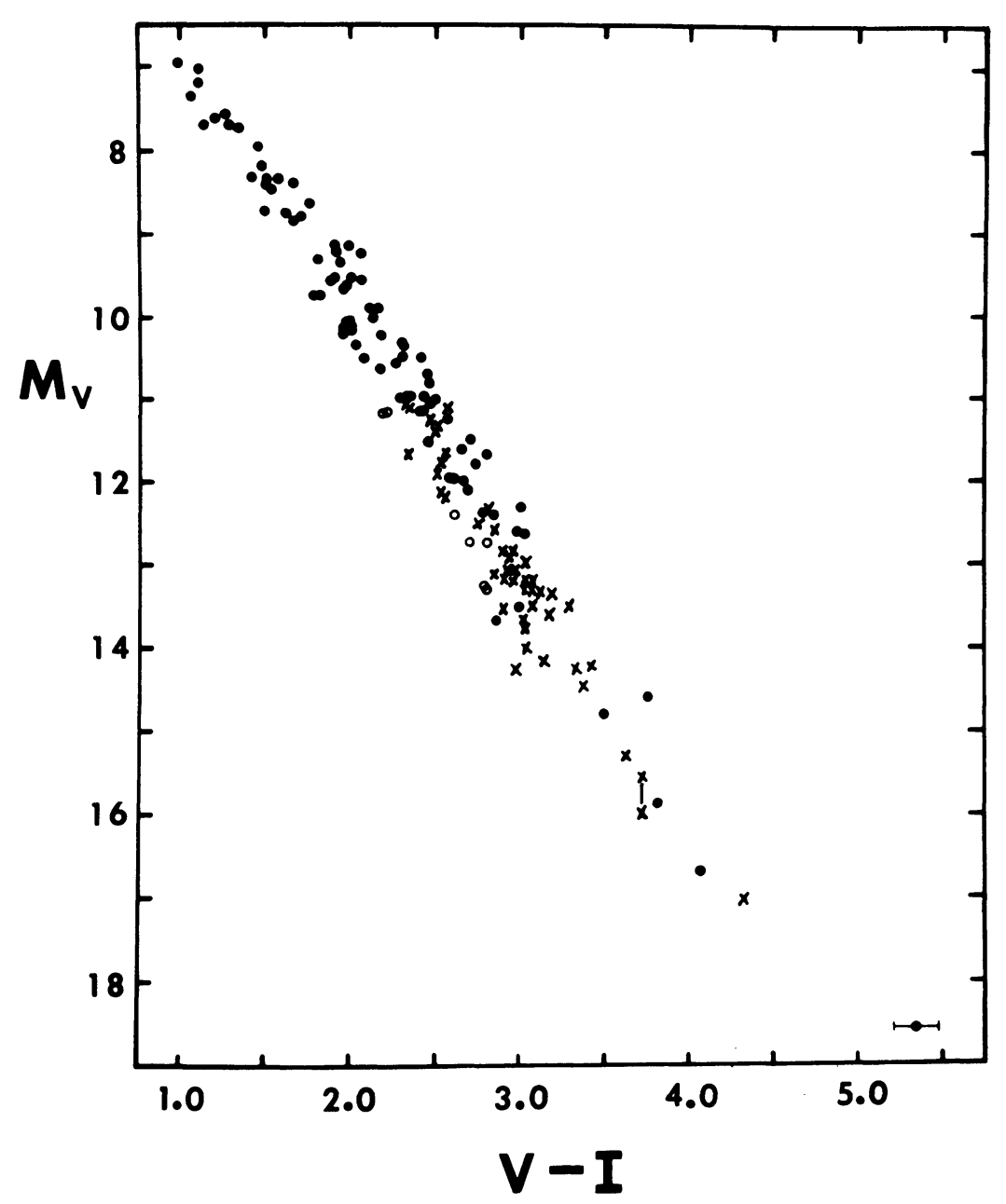

Fig. 3. $M_{v}, V-I$ color magnitude diagram of red dwarfs shown in Figure 2 with additional data $(\times)$ obtained from U.S. Naval Observatory parallax program.

accumulated to date - data which includes near-zero parallaxes for another 10 or so stars which Eggen has suggested as good red subluminous candidates - that (1) slow but steady progress is being made in discovering new candidates for the red degenerate sequence; (2) no parallax sufficiently large to go unchallenged has yet to appear among the USNO program stars to support the contention that stars do populate the region of the $M_{v}$ vs $U-V$ color-magnitude diagram between the subdwarfs and the red degenerate sequence. 


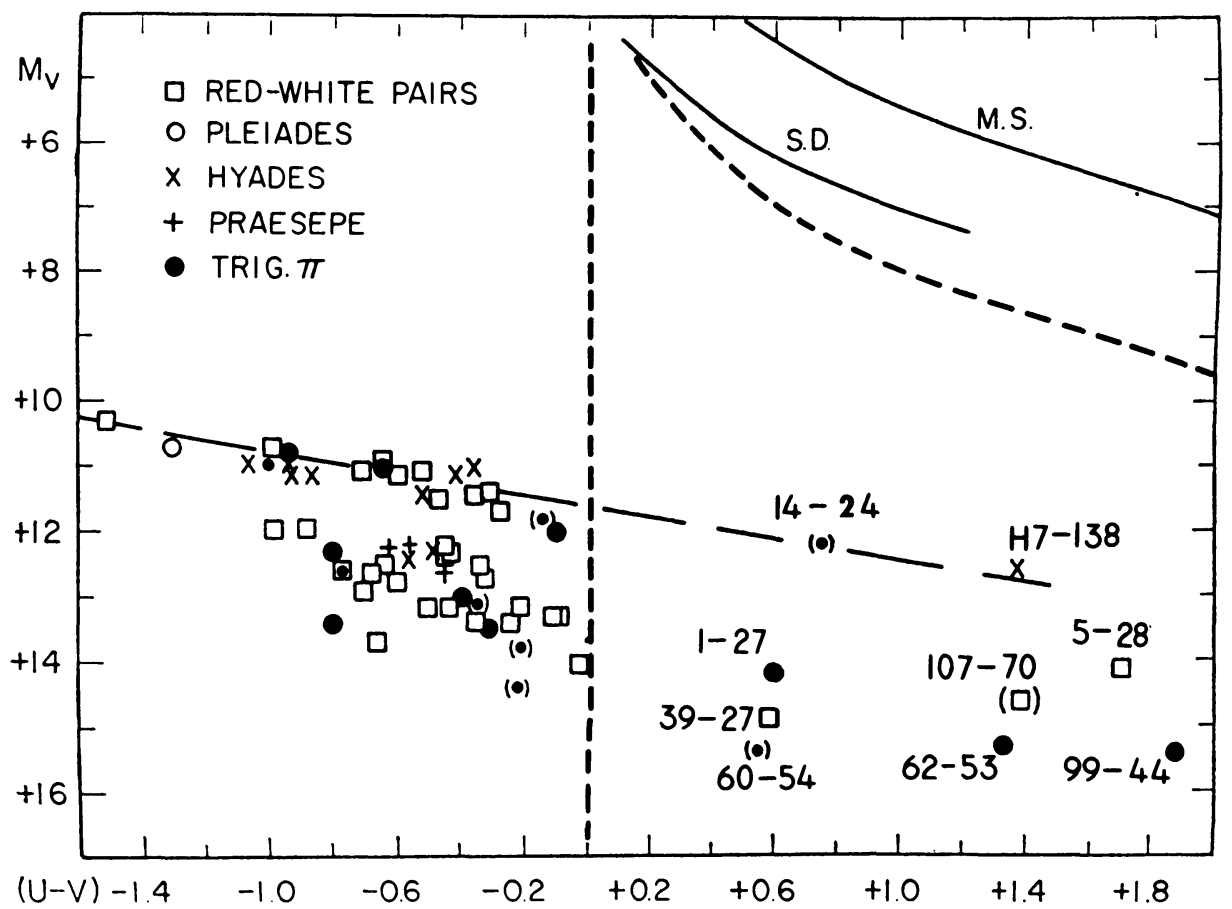

Fig. 4. $M_{v}, U-V$ diagram for white dwarfs, adopted from O. J. Eggen and J. L. Greenstein (Astrophys. $J .141,95,1965)$

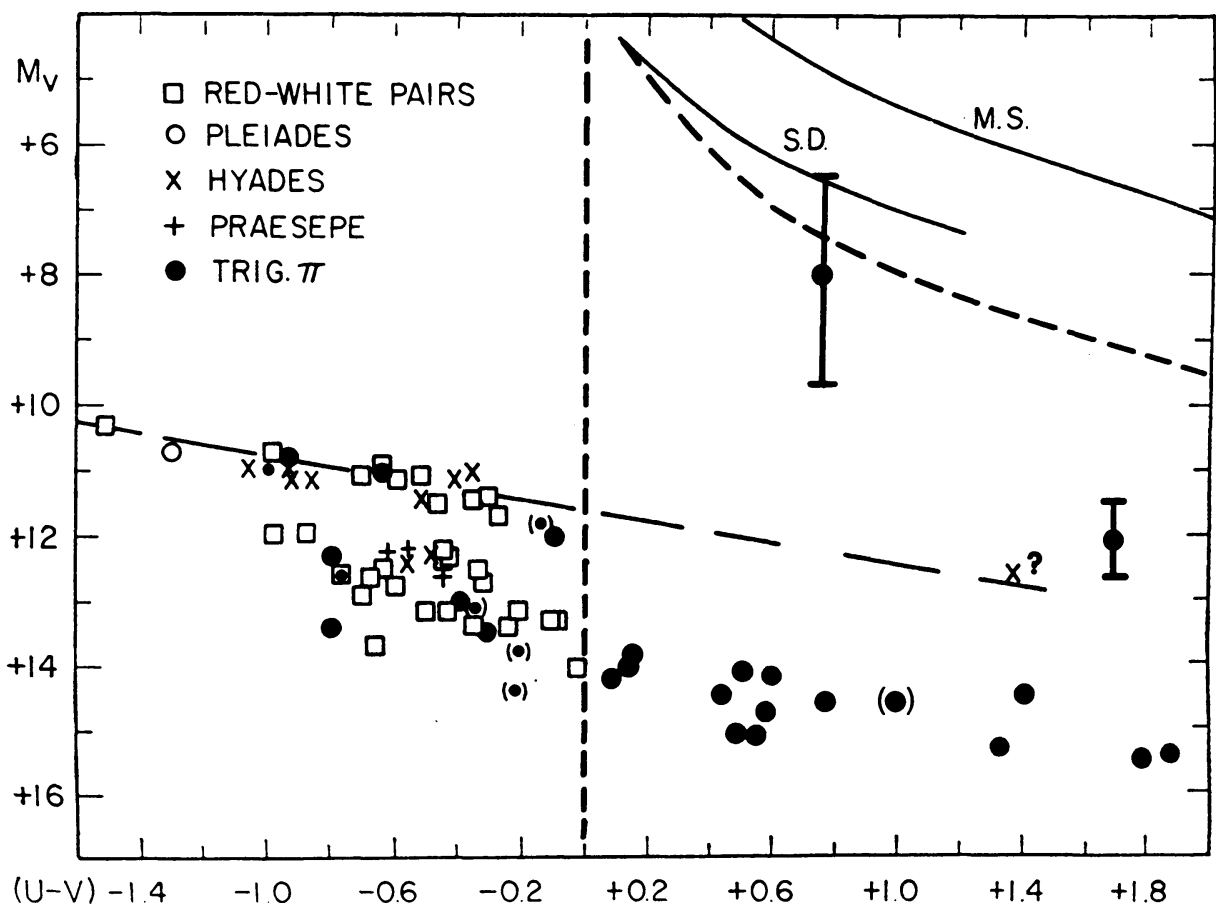

Fig. 5. $M_{v}, U-V$ diagram for white dwarfs, showing revised and new data for the degenerate stars with $U-V>0.0$. 


\section{References}

Dahn, C. C., Behall, A. L., Guetter, H. H., Priser, J. B., Harrington, R. S., Strand, K. Aa., and Riddle, R. K.: 1972, Astrophys. J. 174, L87.

Eggen, O. J.: 1969, Astrophys. J. 157, 287.

Eggen, O. J. and Greenstein, J. L.: 1965, Astrophys. J. 141, 83.

Gliese, W.: 1972, Quart. J. Roy. Astron. Soc. 13, 138.

Greenstein, J. L. and Eggen, O. J.: 1966, Vistas in Astronomy 8, 63.

Hoag, A. A., Priser, J. B., Riddle, R. K., and Christy, J. W. : 1967, Publ. USNO, 2nd Ser. XX, Part II.

Joy, A. H.: 1947, Astrophys. J. 105, 96.

Kron, G. E.: 1958, Publ. Astron. Soc. Pacific 70, 102.

Kron, G. E. and Mayall, N. U.: 1960, Astron. J. 65, 581.

Riddle, R. K.: 1970, Publ. USNO, 2nd Ser. XX, Part IIIA.

Riddle, R. K., Behall, A. L., Guetter, H. H., and Christy, J. W.: 1971, Publ. Astron. Soc. Pacific 83, 210.

Routly, P. M.: 1972, Publ. USNO, 2nd Ser. XX, Part VI.

Strand, K. Aa.: 1971, Publ. USNO, 2nd Ser. XX, Part I.

\section{DISCUSSION}

Harris: You said that the parallax determination levels out after 25 plates; does this also apply to the internal error?

Strand: Yes, but larger than the final value.

Dieckvoss: Do you take double plates against emulsion shift as the refractor people do?

Strand: No, such a procedure would complicate the observing schedule. Since the telescope operation is practically automated the observer is ready with the next plate as soon as the previous plate is completed.

I should mention that the automatic guider guides directly on the polar and declination axes, thus leaving the plate stationary with respect to the optical axis.

Franz: Since a large proper motion is a necessary but not sufficient characteristic for selecting nearby M-dwarfs, would it not be desirable to determine first $V-I$ colours and then, on the basis of proper motions and colours, place suitable stars on a parallax programme. It obviously takes much less time to determine colours than it takes to try to measure (unmeasurable) parallaxes.

Strand: This is the procedure by which M-dwarfs are now added to the programme.

Van Altena: (1) A comparison of 17 stars in common with the Yerkes and USNO parallax programmes yields a systematic error of $+0,0006$ in the sense Yerkes - USNO. The difference in the parallaxes yields an external error $40 \%$ larger than the internal errors if both errors for both observatories are increased by the same amount.

(2) The bifurcation of the white dwarf sequence in the $M_{v}$ vs $U-V$ diagram is a peculiarity of the photometric system. The Stromgren four-color system yields a single well-defined sequence, as shown by Graham and Weidemann.

Strand: Our results indicate that there is also no bifurcation in the $U-V$ diagram for the white dwarfs for $U-V<0.0$. 